\title{
Utilisation de modèles mathématiques multidimensionnels pour l'étude des écoulements dans les digues et barrages en terre
}

\author{
par G. Blanc, Y. Baradat et A. Lagarde \\ Société Franlab
}

La présence d'eau est un facteur important dans la conception d'un certain nombre d'ouvrages de génie civil. Elle agit directement sur la stabilité des talus d'un barrage en terre et sur la mise hors d'eau des grandes fouilles par exemple. Pour l'ingénieur chargé du projet il est donc très important de pouvoir disposer d'un outil de calcul des écoulements de filtration dans son ouvrage ou au voisinage de celui-ci.

L'ensemble des cas pratiques est très varié. Il existe toutefois une particularité commune à tous ces problèmes qui en fait une classe à part dans le domaine de l'hydraulique souterraine : la présence d'une surface libre et éventuellement d'une surface de suintement.

Le problème de la détermination du champ de potentiel de l'écoulement est donc doublé de celui de la détermination

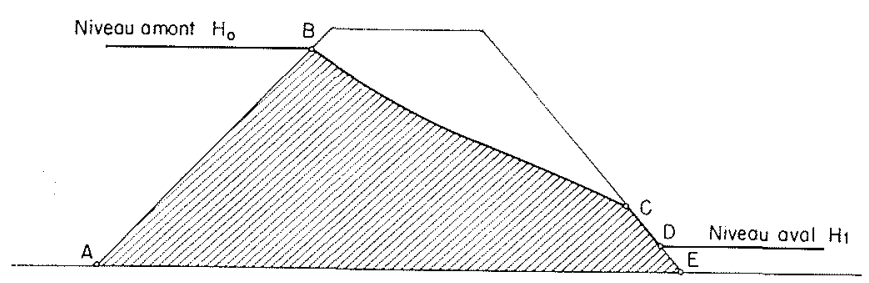

1/ Exemple d'écoulement avec surface libre dans un barrage en terre.
AB Equipotentielle amont $\left(\phi=H_{0}\right)$.
$A E$ Ligne de courant.
ED Equipotentielle aval $\left(\phi=H_{3}\right)$.
DC Surface de suintement $(\phi=z)$.
CB Surface libre $(\phi=z$, ligne de courant).
La partie hachurée du massif est le domaine d'écoulement.

de la forme et de la position de la surface libre qui est une des limites du domaine d'écoulement (fig. 1).

Il est possible d'arriver à la solution de ce double problème par des voies et avec des moyens extrêmement divers : calculs approchés, modèles physiques, modèles analogiques continus ou discrets, modèles mathématiques.

L'utilisation de modèles mathématiques étant la solution qui offre la plus grande souplesse d'emploi, nous avons choisi de développer quelques-unes de ces applications.

Après une description des bases sur lesquelles repose le simulateur d'écoulements et de la méthodologie de détermination de la position de la surface libre, nous examinerons à l'aide d'exemples quelles sont les possibilités des modèles mathématiques et en quoi ils peuvent être utiles à l'ingénieur confronté avec de tels problèmes d'écoulements.

\section{Le simulateur d'écoulements Méthode des éléments finis}

La méthode numérique utilisée par la partie «simulateur d'écoulements 》 des modèles mathématiques présentés ici est la méthode des éléments finis décrite par O.C. Zienkiewicz et Y.K. Chung [1].

Cette méthode est basée sur une formulation tirée du calcul des variations. On peut en effet montrer que lorsque l'intégrale :

$\varepsilon=\int_{\mathrm{V}}\left\{\frac{1}{2}\left[k_{x}\left(\frac{\partial \phi}{\partial x}\right)^{2}+k_{y}\left(\frac{\partial \phi}{\partial y}\right)^{2}+k_{z}\left(\frac{\partial \phi}{\partial z}\right)^{2}\right]-\mathrm{Q} \phi\right\} d \mathrm{~V}$ 
prise dans tout le domaine $\mathrm{V}$ de l'écoulement peut être minimisée, cette minimisation est une écriture équivalente à la formulation différentielle d'un phénomène permanent, soit :

$$
\begin{aligned}
\frac{\partial}{\partial x}\left(k_{i} \frac{\partial \phi^{\prime}}{\partial x}\right) & +\frac{\partial}{\partial y}\left(k_{y} \frac{\partial \phi^{\prime}}{\partial y}\right) \\
& +\frac{\partial}{\partial z}\left(k_{z} \frac{\partial \phi^{\prime}}{\partial z}\right)+\mathrm{Q}=0
\end{aligned}
$$

avec :

$\phi^{\prime}=(p / \rho g)+z$ le potentiel moteur de l'écoulement;

$p$ la pression;

$\rho$ la masse volumique du liquide;

$x, y, z$ les coordonnées;

$k_{x}, k_{y}, k_{z}$ les composantes principales du tenseur de perméabilité dont les axes sont ici supposés parallèles aux axes de coordonnées;

Q la densité du flux prélevée ou injectée.

Si de plus les fonctions $\phi$ et $\phi^{\prime}$ obéissent aux mêmes conditions aux limites, on a identité formelle des deux formulations et :

$$
\phi \equiv \phi^{\prime}
$$

Comme il ne saurait être question de rechercher analytiquement une fonction $\phi$ qui minimise $\varepsilon$ et qui satisfasse les conditions aux limites, on a recours à une division du domaine en un certain nombre d'éléments finis et c'est à l'intérieur de chacun de ces éléments finis que l'on applique le processus de minimisation.

Sur le plan pratique, la mise en œuvre de la méthode est effectuée de la façon suivante :

1. - On se fixe la forme géométrique des éléments (fig. 2). Cette forme est définie par un certain nombre $n$ de nœuds.

2. - Dans chaque élément on choisit une fonction d'interpolation du potentiel. Cette fonction s'appuie sur les valeurs nodales $\phi_{i}, \phi_{j} \ldots \phi_{n}$ que prend $\phi$ en chacun des $n$ nœuds de l'élément, par exemple :

$$
\phi=\phi_{\mathrm{i}} \cdot \mathrm{N}_{\mathrm{i}}+\phi_{j} \cdot \mathrm{N}_{j}+\ldots \phi_{n} \cdot \mathrm{N}_{n}
$$

avec $\mathrm{N}_{i}, \mathrm{~N}_{j} \ldots \mathrm{N}_{n}$ des fonctions de $x_{i}, y_{i}, z_{i} \ldots x_{n}, y_{n}$, $z_{n}$ et des coordonnées du point courant $x, y, z$ qui déterminent le type d'interpolation choisi:

On trouvera dans l'ouvrage de O.C. Zienkiewicz et $Y . K$. Chung [1] les conditions auxquelles les fonctions de base $N$ doivent obéir pour que la convergence de la méthode soit acquise.

3. - Ceci étant posé, on peut évaluer les dérivées partielles de $\varepsilon$ par rapport à $\phi_{i}$ dans l'élément $e$ que l'on notera $\partial \varepsilon^{o} / \partial \phi_{i}$. Tous calculs faits on aboutit à :

$$
\frac{\partial \varepsilon^{e}}{\partial \phi_{i}}=b_{i}^{e}+a_{i i}^{e} \phi_{i}+a^{e}{ }_{i j} \phi_{j}+\ldots+a_{i n}^{e} \phi_{n}
$$

c'est-à-dire à une relation linéaire en $\phi_{i}, \phi_{j} \ldots \phi_{n}$ dans laquelle les coefficients ont pour expression :

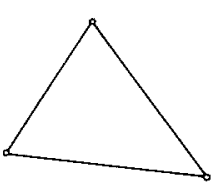

3 degrés de liberté

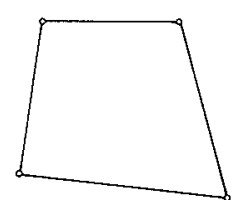

4 degrés de liberté - ELLEMENTS PLANS

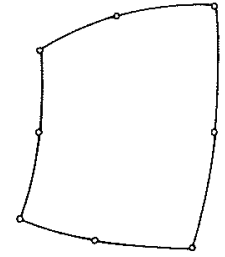

8 degrés de liberté
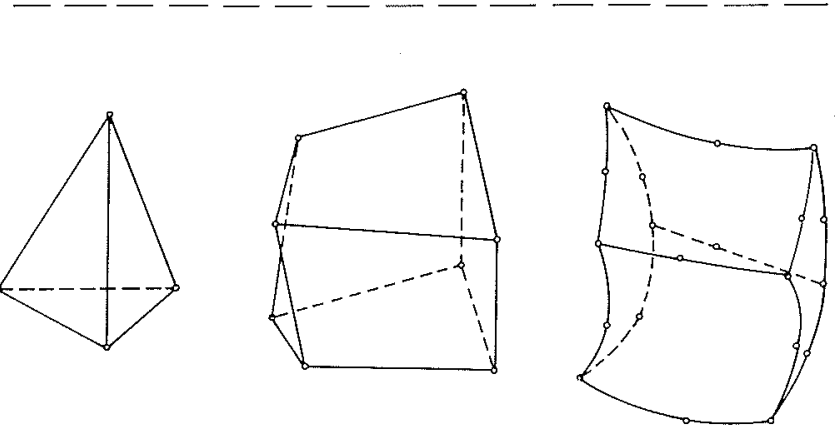

4 degrés de liberté

8 degrés de liberté

20 degrés de liberté

- ÉLÉMENTS TRIDIMENSIONNELS -

2/ Exemples d'éléments.

$a_{i j}^{c}=\int_{\mathrm{V} v}\left(\begin{array}{r}k_{x} \frac{\partial \mathrm{N}_{i}}{\partial x} \frac{\partial \mathrm{N}_{i}}{\partial x}+k_{y j} \frac{\partial \mathrm{N}_{i}}{\partial y} \frac{\partial \mathrm{N}_{i}}{\partial y} \\ +k_{z} \frac{\partial \mathrm{N}_{i}}{\partial z} \frac{\partial \mathrm{N}_{i}}{\partial z}\end{array}\right) d \mathrm{~V}$

$b_{i}^{c}=-\int_{\mathrm{Ve}} \mathrm{Q} \mathrm{N}_{i} d \mathrm{~V}$

et sont calculables à partir des expressions de $\mathrm{N}_{i}, \mathrm{~N}_{j}, \ldots \mathrm{N}_{\mu}$ et de la forme des éléments.

4. - La sommation de l'ensemble des contributions de chacun des éléments au processus de minimisation conduit à la relation matricielle :

$$
[\mathrm{A}] \cdot[\phi]=[\mathrm{B}]
$$

dans laquelle [A] est la matrice carré d'ordre $M$ (si $M$ est le nombre total de nœuds de l'ensemble des éléments) obtenue à l'issue du processus, $[\phi]$ est la matrice colonne qui contient l'ensemble des $M$ valeurs nodales de la fonction et [B] la matrice second membre qui prend en compte outre le terme $Q$ de l'équation (2), les diverses conditions aux limites dont nous n'avons pas tenu compte dans cette description sommaire de la méthode.

La relation (8) montre que l'on peut donc calculer l'ensemble des valeurs nodales de la fonction potentiel en résolvant le système linéaire (8) et disposer ainsi de la solution du champ potentiel recherché. 


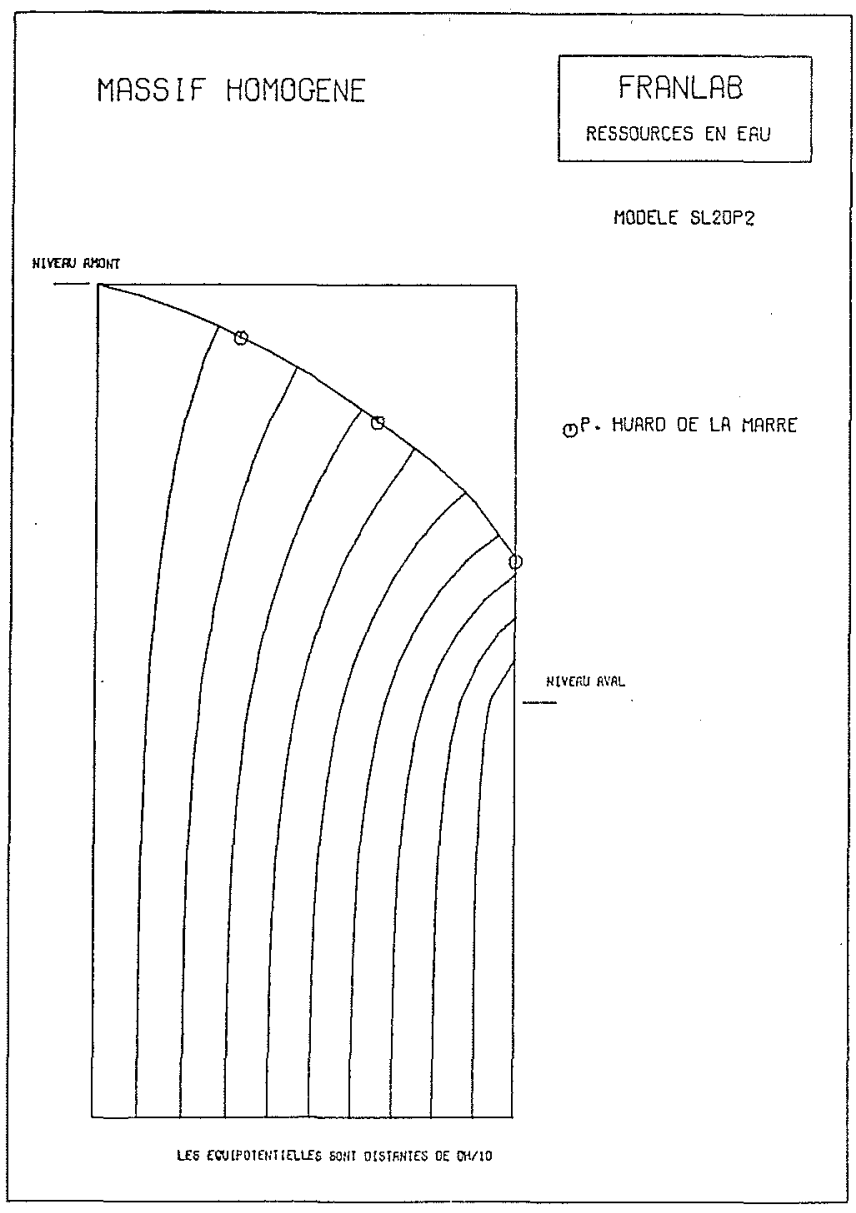

3/

En examinant quelques-uns des éléments que la méthode permet d'utiliser, et dont certains ont été effectivement utilisés pour traiter les exemples décrits plus loin, on conçoit que cette méthode soit particulièrement adaptée aux calculs d'écoulements avec surface libre. Elle permet en particulier de faire coïncider parfaitement les limites du domaine de simulation avec celles du domaine d'écoulement et avec la surface libre.

\section{Détermination de la surface libre}

La fonction potentiel obéit à deux types de conditions aux limites le long de la surface libre (fig. 1):

1. - Pression égale à la pression atmosphérique, ce qui entraîne :

$$
\phi=\frac{p_{a}}{\rho g}+z
$$

et

$$
\phi=z
$$

lorsque la pression atmosphérique est prise comme référence de mesure des pressions $\left(p_{a}=0\right)$.

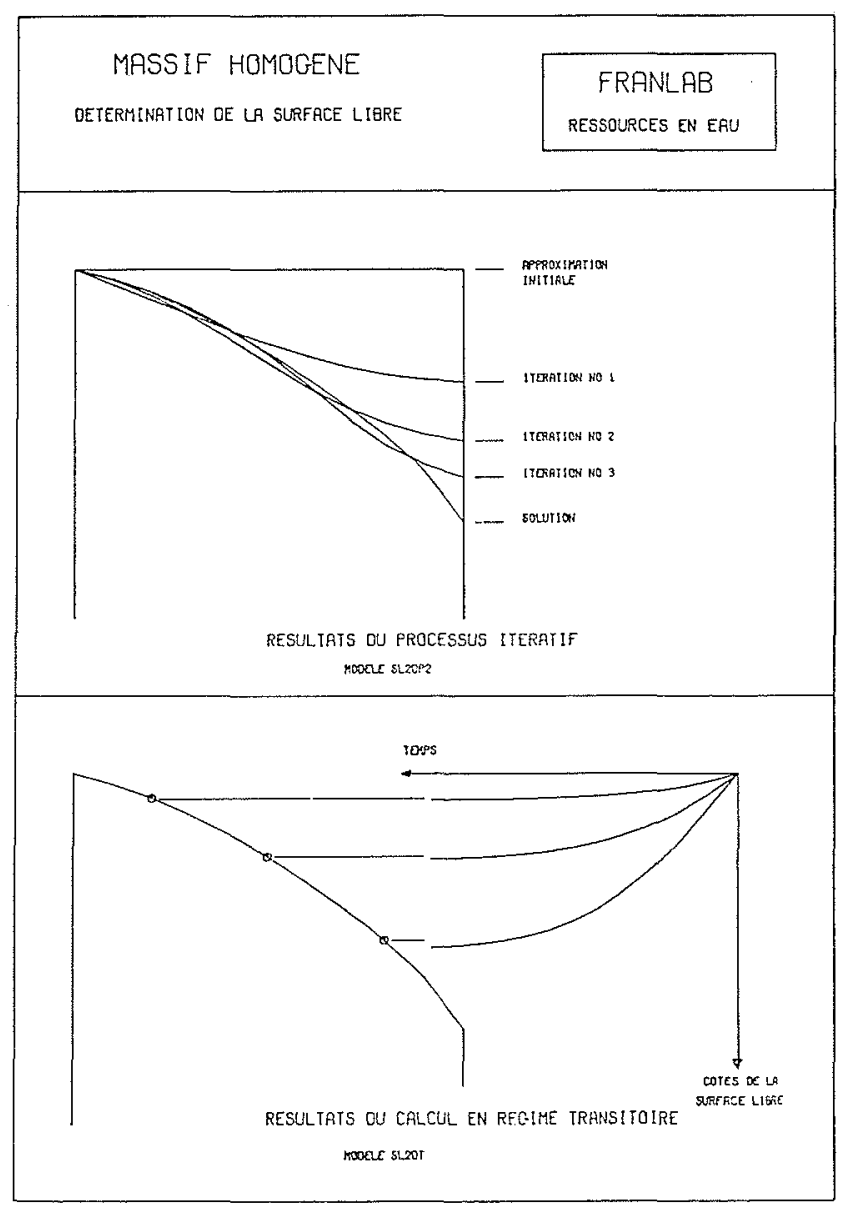

$4 /$

2. - Flux nul ou fixé par les conditions d'alimentation de la surface libre.

Il est bien évident que ces deux conditions découlent de l'assimilation de la surface libre à une séparation abrupte entre la partie saturée du massif et l'air. Nous verrons plus loin qu'il est possible de prendre en compte les phénomènes capillaires qui transforment cette séparation en une zone dans laquelle la saturation en eau varie de façon continue.

Sur le plan pratique, nous avons étudié trois types de détermination des surfaces libres en régime permanent.

Obtention de la surface libre par approximations successives.

Cette méthode est bien connue des utilisateurs des modèles analogiques et on en trouvera l'exposé dans les travaux de P. Huard de la Marre [2]. Elle consiste à améliorer pas à pas la position de la surface libre en fonction du champ de pression obtenu à chaque approximation.

La figure 4 regroupe quelques itérations de ce processus dans le cas d'une digue perméable homogène à parois verticales. La figure 3 montre que la solution ainsi obtenue est d'excellente qualité par comparaison avec les résultats d'un modèle analogique.

Obtention de la surface libre en permanent limite d'un régime transitoire.

La figure 4 illustre l'utilisation d'un modèle mathématique transitoire (modèle SL2DT) pour la recherche du 


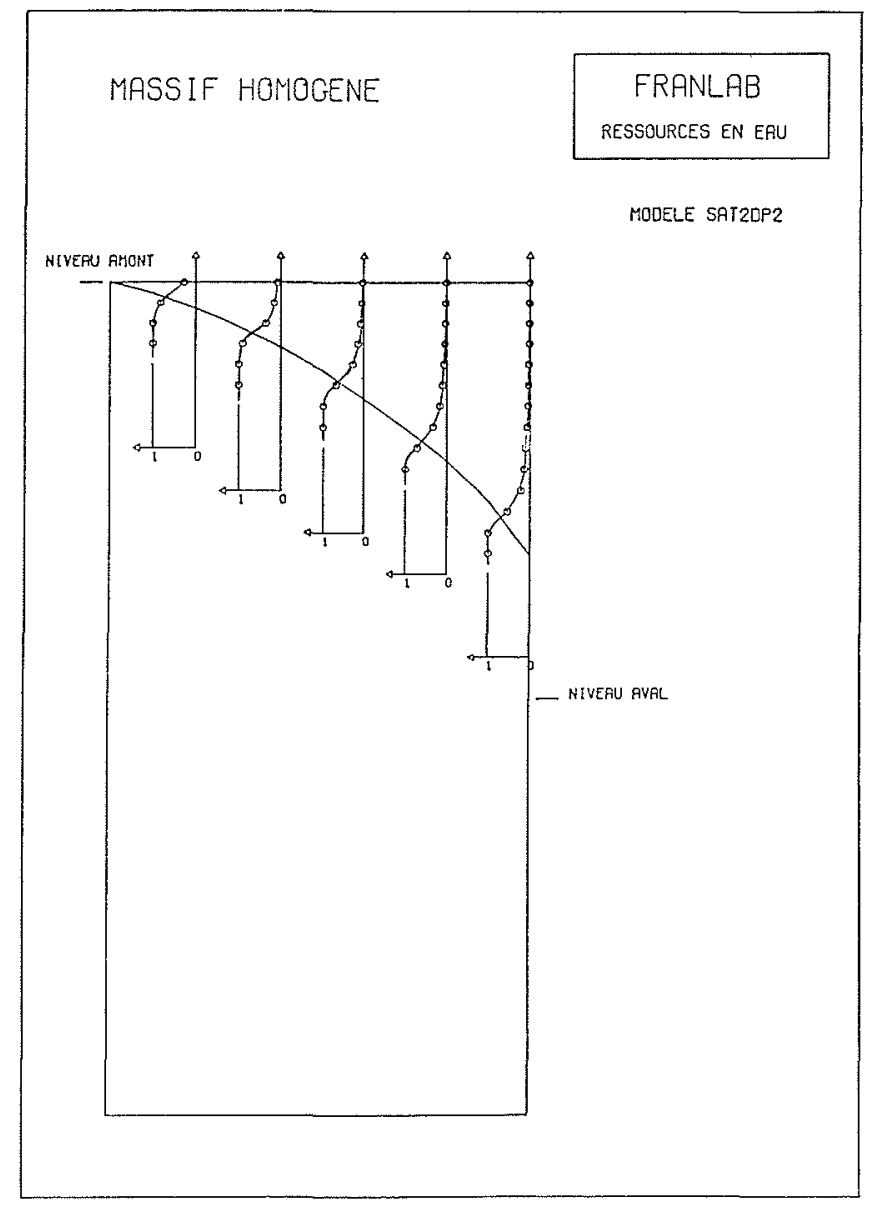

5/

6/

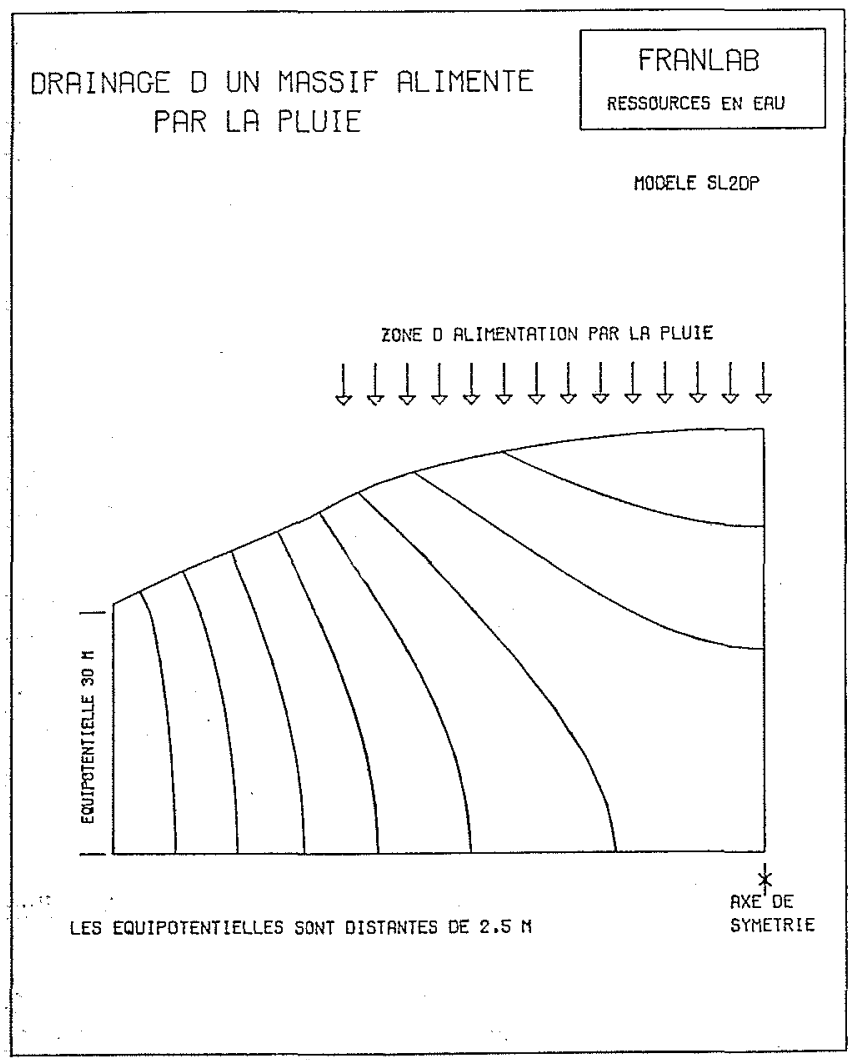

régime permanent de l'exemple déjà décrit. A l'instant initial le massif est totalement noyé et l'on ramène brusquement le niveau aval à sa position finale. Le régime transitoire fictif obtenu tend vers un régime stable proche du précédent calculé par approximations successives.

Prise en compte des phénomènes capillaires (modèle $S A T 2 D P 2)$.

Le même exemple a été encore une fois utilisé mais cette fois-ci en tenant compte des phénomènes capillaires. On ne peut plus parler de surface libre dans ce cas mais on constatera sur la figure 5 que la position de la zone de transition entre la saturation maximale et la saturation minimale en eau diffère peu de la surface libre calculée. La section d'écoulement est toutefois légèrement ouverte par le phénomène de succion, le débit qui transite à travers le massif poreux est alors légèrement supérieur au précédent.

La perméabilité étant une fonction de la saturation et donc du potentiel dans le massif, ce problème non linéaire a été résolu par approximations successives en tant que régime permanent. Comme dans l'hypothèse de la surface libre abrupte il serait également possible dans ce cas de concevoir la solution du régime permanent comme limite d'un régime transitoire fictif.

7/
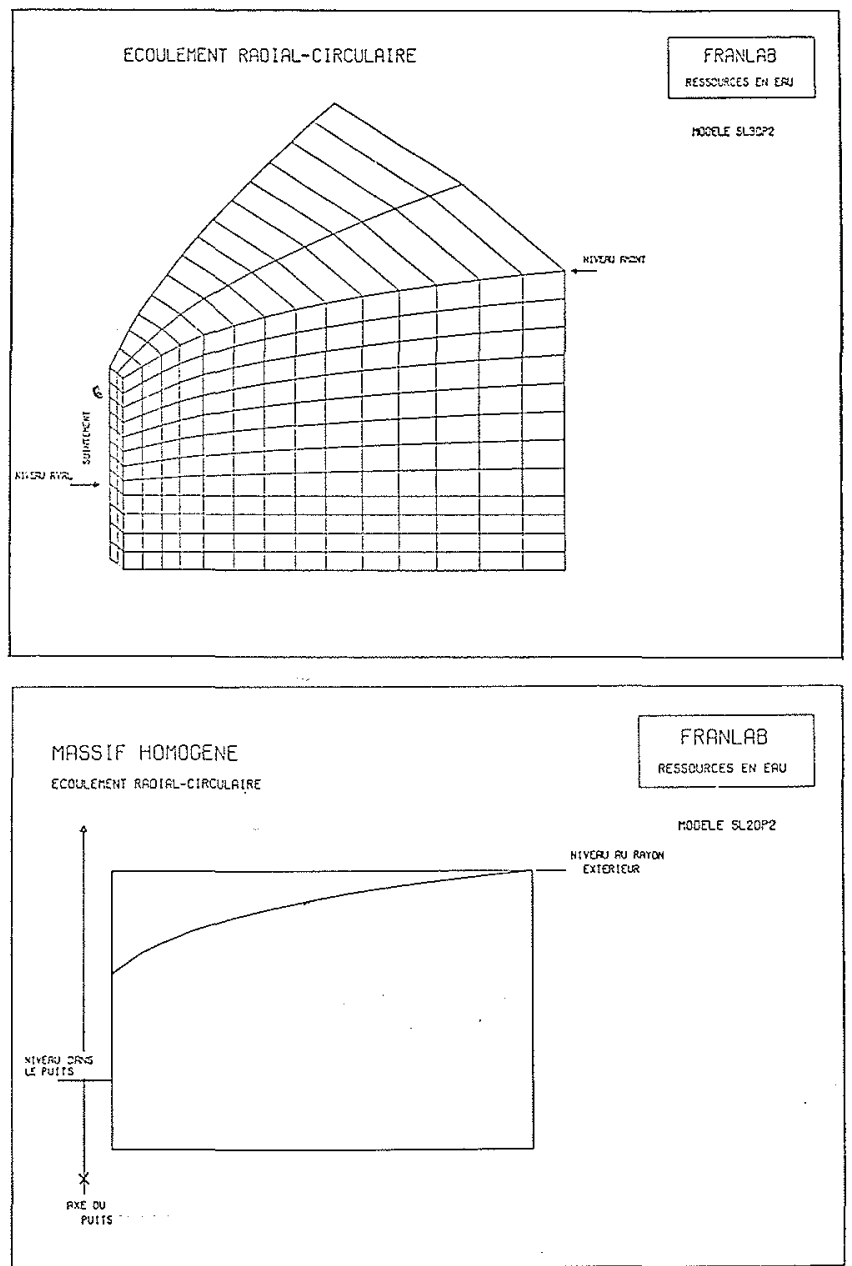


\section{Utilisation des modèles mathématiques}

Le domaine d'utilisation des modèles mathématiques est très étendu. A l'aide de quatre exemples nous allons examiner quel peut être leur apport dans des cas aussi divers que l'hydraulique agricole, l'hydraulique des puits, les problèmes de stabilité des digues et barrages en terre, le calcul des débits de fuite.

\section{Hydraulique agricole.}

La figure 6 décrit le calcul du drainage d'un sol partiellement alimenté par la pluie à l'aide de deux tranchées parallèles. Ce schéma peut être aisément modifié pour des études d'efficacité de drains de formes et d'éloignements différents. Le modèle mathématique permet également de prendre en compte d'autres paramètres comme l'anisotropie du sol, la présence d'hétérogénéités, etc. L'influence des phénomènes capillaires peut être également étudiée à l'aide d'un modèle mathématique décrit plus haut (mođèle SAT2DP2, fig. 5).

\section{Hydraulique des puits.}

Il n'est pas toujours possible d'interpréter un essai de pompage à l'aide des résultats de la littérature spécialisée. Le modèle mathématique permet alors de simuler le programme de pompage et d'ajuster les paramètres de la nappe en comparant les mesures et les résultats du calcul.

L'exemple de la figure 7 a été résolu à l'aide d'un modèle mathématique radial-circulaire et également à l'aide d'un modèle mathématique tridimensionnel.

Dans ce domaine, l'intérêt du modèle tridimensionnel est de permettre l'étude d'essais multiples dans des aquifères à caractéristiques quelconques et pour des conditions aux limites également quelconques.

\section{Stabilité des barrages en terre.}

La position de la surface libre et la répartition des pressions interstitielles dans la partie saturée d'un barrage en terre sont deux éléments déterminants du calcul de stabilité de cet ouvrage. L'étude doit être faite : dans les conditions normales d'exploitation (retenue à la cote maximum) pour examiner les conditions de stabilité du parement aval; dans le cas d'une vidange rapide pour examiner les conditions de stabilité du parement amont ainsi que les dommages causés à l'ouvrage par la désaturation; et en cours de construction.

Les résultats d'un exemple de calcul hydraulique pour un barrage en terre à noyau reposant sur une fondation légèrement perméable sont donnés sur la figure 8. Le programme fournit toutes les données nécessaires aux calculs de stabilité (partie en eau du massif, pressions locales dues à l'écoulement, vitesses locales dans le massif et sur les surfaces de sortie). L'effet de paramètres comme la perméabilité et le degré d'anisotropie des différentes parties du massif peut être facilement étudié sans aucune modification du programme lui-même.

Le problème du dimensionnement des rideaux étanches utilisés pour minimiser les phénomènes de renard à l'aval des retenues est également un cas où les modèles mathématiques peuvent être utilisés avec profit.

Enfin il est également possible de reprendre tous ces calculs pour étudier l'influence de la troisième dimension des massifs dans certains cas bien précis où elle ne peut être négligée (paragraphe suivant).

\section{Estimations des débils de fuite.}

Le problème décrit sur la figure 9 est typiquement tridimensionnel. Il s'agit en effet d'évaluer le débit qui fuit par les berges perméables d'une retenue elle-même imperméable. A partir de ces résultats il est possible de prendre des décisions pour limiter ce débit et de tester leur validité. Là encore, on pourrait, à l'aide du même modèle, traiter des cas plus complexes dans lesquels le barrage et ses fondations seraient également le siège d'écoulements.

Le calcul des débits de fuite est aussi un paramètre important pour la conduite de l'exécution de grandes fouilles dans des nappes aquifères ou dans l'évaluation d'exhaures miniers.

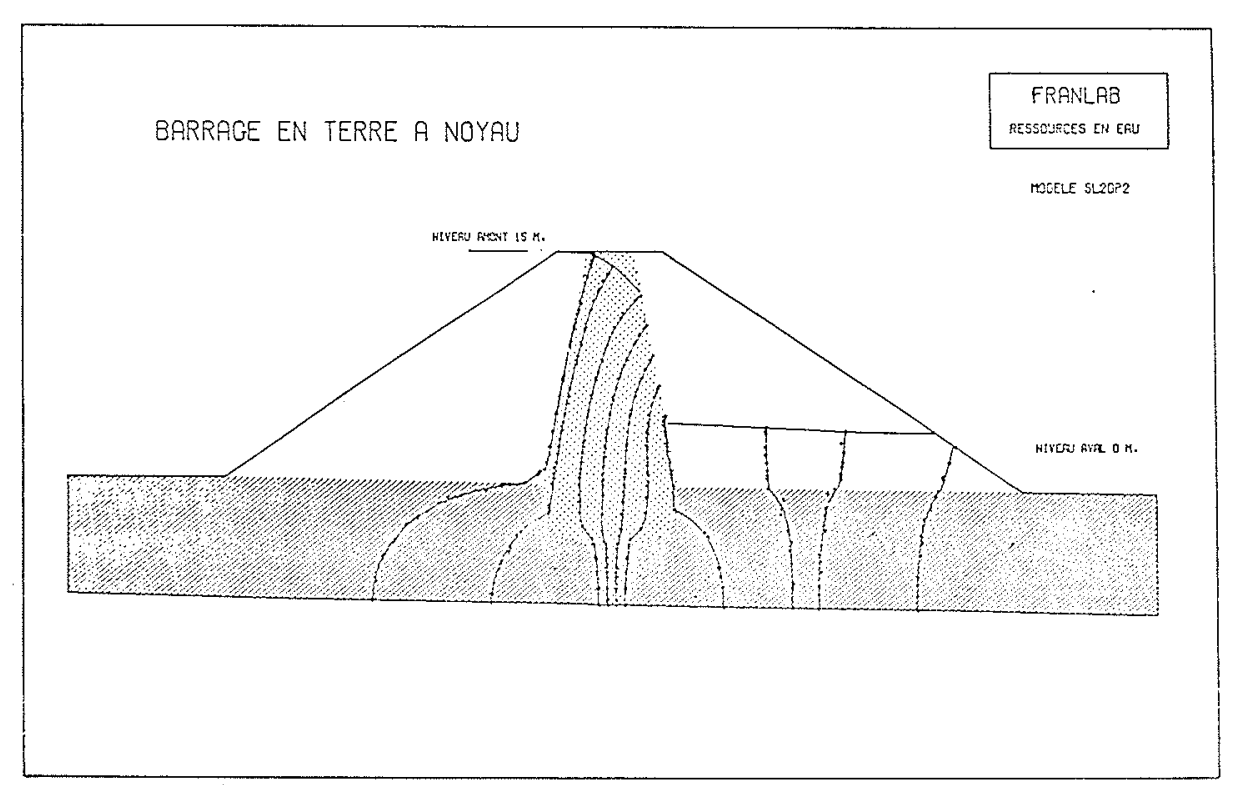




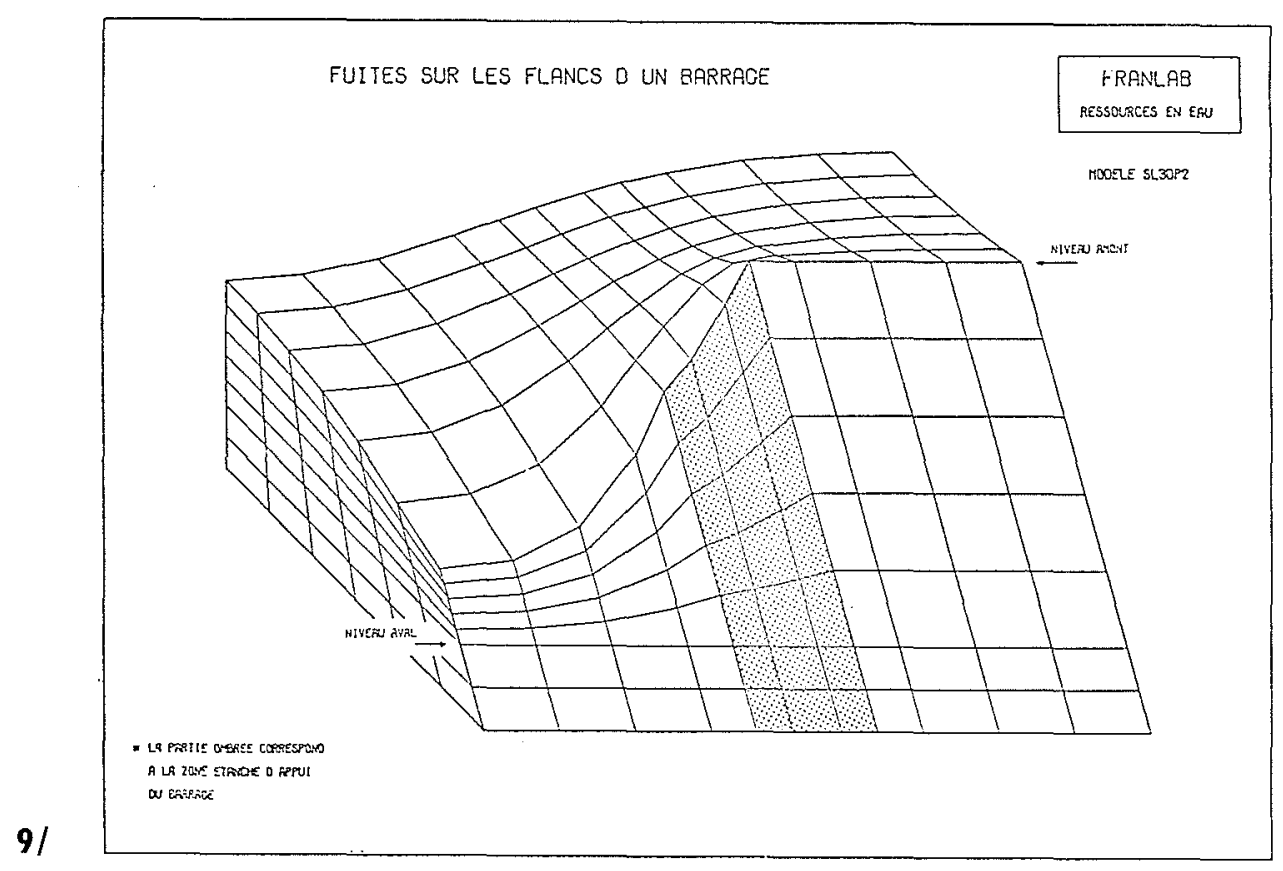

\section{Conclusions}

Les quelques exemples qui viennent d'être décrits montrent que les modèles mathématiques peuvent rendre des services importants dans un grand nombre d'études de génie civil ou d'hydraulique agricole.

L'apport du modèle mathématique dans l'arsenal des outils mis à la disposition de l'ingénieur réside dans l'étendue incomparable des possibilités qu'il offre à son utilisateur. Les résultats qu'il fournit sont précis et ont l'avantage de pouvoir être traités dès leur édition par une chaîne de programmes auxiliaires (à titre d'exemple, on remarquera que les figures 3 à 9 ont été éditées par un traceur Calcomp). L'introduction des données peut être facilitée et rendue plus précise à l'aide d'appareils de digitalisation qui repèrent les coordonnées nodales sur un plan du maillage. D'autre part, l'apparition de puissants ordinateurs scientifiques tel le CDC 7600 permet de s'affranchir des problè- mes inhérents aux méthodes de résolution des grands systèmes d'équations tout en conservant leurs performances aux modèles mathématiques.

Toutefois, il ne faut voir dans ces modèles que des outils mis à notre disposition dont l'utilisation sera d'autant plus profitable qu'elle aura été précédée d'un travail de réflexion et de synthèse au stade de la préparation de l'étude.

\section{Références}

[1] Zienkiewicz (O.C.) et Chung (Y.K.). - The finite element method in structural and continum mechanics. Mc Graw-Hill, Londres (1970).

[2] Huard de LA Marre (P.). - Résolution de problèmes d'infiltration à surface libre au moyen d'analogies électriques. Publications Scientifiques et Techniques du Ministère de l'Air, $\mathrm{n}^{\circ} 340(1958)$.

\section{Discussion}

Présidents: MM. G. Kovacs et P. HabIB

M. le Président Habib temercie M. Blanc de son clair exposé et onvre la discussion.

M. Verruist (Université Technique de Delft; Pays-Bas) intervient en ces termes:

Je suis complètement d'accord avec M. BLANC lorsqu'il estime que la méthode des éléments finis est à présent la méthode la meilleure pour l'analyse des écoulements en milieu poreux, surtout dans le cas où il existe une surface libre.

Je n'ai qu'une petite question à lui poser : «Quelles sont les équations que vous avez utilisées dans la zône non saturée; il me semble que l'on pourrait avoir des difficultés du fait de la non-linéarité des équations, mise en évidence par M. VACHAUD ?»

Le modèle utilise les équations que $M$. Vachaud a présentées dans son mémoire; ce sont, en effet, des équations non-linéaires, répond $\mathrm{M}$. BLANC.

M. Dupas youdrait connaitre le nombre d'itérations nécessaires pour obtenir, en pratique, la solution. Quelle est la différence entre les réseaux d'écoulement obtenus par deux approximations 
se succédant? L'efort et le coût supplémentaires exigés par la deuxième approximation sont-ils justifiés en pratique?

Cela dépend de ce que lion demande au modele mathématique répond $M$. Buanc. Dans l'exemple ciassique de la digue à parois verticales, le débit de filtration à travers la digue est pratiquement exact à la première itération di processus, par contre cinq itérations environ sont nécessaires pour obtenir la surface libre avec une précision suffisante. En termes de coût, on peut dire que, dan la majorité des cas, quelques itérations supplémentaires n'affectent pas sensiblement le cout total d'une étude, sauf́ lorsque des modèles tridimensionnels sont utilisés falans ce dernier cas, ha construction et la résolution đu système linéare des équations nodales sont des opérations plus longues à exćcuter).

Sur une question de M. Je Professeur Thukrot, M. BLANC précise

Il existe deux stratégies possibles en matière d'utilisation des modèles mathématiques. On peut essayer de concevoir un modèle généra! ou bien avoir un arsonal de modeles que l'on reprend chaque fois pour fabriquer un outil adapté à une étude précise. En ce qui conceme plus particulierement les modeles i surface libre, il parât extremement difficile de concevoi un modele tridimensionmel genérat; en bidimensionnel c'est plus facile.

Alt sujet de la denxieme partie de la question de M. lo protesseur ThrRrot, M. BLANC ajoute:

11 n'est possible de sauvegarder une partie des calculs de fabrication de la matrice du systeme linéaire que lorsque la technique de modification du maillage à chaque iteration s'y prete.

Lorsque le maillage est dilaté on contracte a la fo de chaque itération, cette sauvegarde est exclue sauf si une partie du maillage est fixe.

Personnellement, nous utilisons en bidimensionnel une technique qui consiste à ne modifier que les eléments qui sont au voisinage de la surface libre, les autres tant inchanges. Dans ce cas on peut alors envisager de ne modifier qu'une faible partic de la matrice du système linéaire à chaque itération et économiser aussi un certain volume de calcul.

M. le Frésident souligne - daccord avec M. Buanc - que lo nombre d'itérations nécessaires riest pas un critère déterminant an les calculs a l'ordinateur; ces iterations demandent quelques se. condes d'ordinateur qu'il ne vaut pas he peine de chercher économiser car, dans la majorité des cas, leur cont est négligeable par rapport à celui de la préparation du "passare en machne de l'ensemble du problème.

Concemant le nombre ditérations nécessaires pour obtenit un évaluation satisfaisante des pressions et de la ligne de surface libre, M. RuefF donne les résultats obtenus sur un modele d'éléments finis construit par SOGREAH pour la recherche directe des pression intersticielles et de la surface libre de régime permanent pron modèle, d'une conception différente de celle indiqué par MM Bive, Baradat et LAGarde, les pressions et surface libre démitives ont été en pratique trouvées automatiquement en trois itérations (fienxième surface libre suivant la surface arbitraire de départ).

M. P. UNGemach (Service Géologique d"Alsace et Lorraine, B.R.G.M.) intervient dans le but de prolonger la communication de M. Blanc par une illustration des possibilites des techniques de simulation sur modeles dais la résolution d'un cas concret d'aménagement de génie civil Le caractère probatoire de l'étude n'exclut nullement une critique permettant d'améliorer à l'avenir la représentativité de tels modeles.
Le problème, traité par le laboratoire dhydrodynamique du S.G.A.-B.R.G.M. à la demande de la Région d'Equipement $\mathrm{Hy}$ draulique Nord de lElectricité de France, consistait à rechercher un optimum de la mise hors d'eau des fouilles usine-écluses de la future chute de Gambsheim, neuvieme bief de l'aménagement hydroélectrique du Rbin. Les caractéristiques de l'aménagement, implanté dans des terrains alluvionaires fortement perméables, à proximité immédiate d'un feuve de débit moyen de $1250 \mathrm{~m}^{3} / \mathrm{s}$ (débit de crue : $4000 \mathrm{~m}^{3} / \mathrm{s}$ ) localement peu colmaté, imposaient des précautions particulières dans le dimensionnement du dispositif de drainage.

Compte tenu de l'expérience acquise par le S.G.A.L.-B.R.G.M. dans le domaine des modèles analogiques électriques, le problème se présentait dans des conditions favorables pour être simulé par un modèle évolué de type réseal de résistance, de près de 50000 mailles.

Lhypothèse était celle d'écoulements tridimensionnels stationnaires en régime non linéaire à surface libre; les caractéristiques du maillage variaient fortement dans les trois directions de l'espace.

L'étude comporte dely phases distinctes:

- Elalonnage du modéle sur le site du bief précédent (chute de Strasbourg): recherche du degré d'anisotropie et de la perméabilite horizontale moyens du gisement alluvionnaire.

- Recherche doptimum: les caractéustiques hyorodynamiques moyennes des alluvions, deduites de létalonnage, sont reconduites sur to site du bief futur on le disposinf de mise hors d'eau (parois monlées, drains, rigoles de colature, souilles de pompage, puits de décompression) est optimisé au regard des coúts de mise en auve et dexplotation. Un compromis est réalisé, pour une sécurité nonnale de 1.2 , entre les conts fe pompage et dapprofondissement des volles étanches.

Ze modele, construit aveo des circuits miniaturisés, a été concu de facon a simuler les dew sites de centrales, pour une anisotropie susceptible de varier entre 50 et 200 . En particulier, la recherche simmianee, en phase de calage, des perméabitites horizontale et verticale dans thypothese tune distribution whiorne dans le champ, a été réalisée, sans modification des composants du réseas, par simple dilatation d'échelle géométrique et variation des rapports d'analogia.

Les indications du modele ont pu, fat assez rare dans la profession, ête conrontees aux observations du chantier. Les débits dexhaure calculés sur modèle se son révéles, en certains endroits, tres infrieurs a la réalité. Ia raison doit en être recherchée, moins dans le modele lui-même, Gui dispose d'une technologie opérationnelle, que dans les critères de représentation du milieu physique. Fis: varticutier, ladoption dun aquifere monocouche equivalent et lextapolation des perméabilités du hutième au neuvieme bief se sont révélées insufisantes. 11 s'agit donc essentiallement dun probleme de reconnaissance in-situ et de collecte d'observations quantifables. Une nouvelio méthodologie d'opérations a été ains proposée sur le site du futur aménagement a l'aval de Gambsheim, lefort portant sur lexploration du milieu naturel et I'identifica. ton de son feulletage atu sens de sa géométrie et de ses propriétés hydrodyramiques. Des modeles mathématiques, à symétries variés et a utilisation ponctuele, permettent d'ameliorer l'interpretation des cssals de pompage et des fuctuations de piézométrie à proximite du fleuve, et de compléter les informations géologiques, granulomé triques et les essais de cavité de type Lefranc.

en conclusion, de tels modeles permettent de traiter des problemes réels complexes, liés à une exécution rationnelle et sôre d'aménagements coûteux, au sens de la simulation et de la rechercho dune représentation fidelc du milieu en placo.

M. Le Président Habig clót la discussion en remerciant tous les intervenants et annonce une suspension de séance de dix minutes. 